\title{
DESENVOLVIMENTO DE SOFTWARE EDUCACIONAL COMO FERRAMENTA DE ENSINO NA ENGENHARIA CIVIL - LAJE SIMPLES MACIÇA DE CONCRETO ARMADO
}

\author{
Fernando de Andrade Dolabella \\ Bacharel em Engenharia Civil no Centro Federal de Educação Tecnológica Celso Suckow da \\ Fonseca (CEFET/RJ), Rio de Janeiro, RJ, Brasil \\ fernando.dolabella@aluno.cefet-rj.br
}

Ricardo Rodrigues de Araujo

Professor do Centro Federal de Educação Tecnológica Celso Suckow da Fonseca (CEFET/RJ), Rio de Janeiro, RJ, Brasil

ricardo.araujo@cefet-rj.br

\section{RESUMO}

Este projeto trata do desenvolvimento de software capaz de realizar o dimensionamento de lajes maciças simples, por meio do processamento de dados em uma aplicação web. O mesmo foi criado devido à uma necessidade dos alunos da engenharia civil de uma forma eficiente de estudar e corrigir seus exercícios, que se propõe neste programa. Com a demonstração, por meio de resolução de um exercício típico, foi possível observar a eficácia com que o programa exerce sua função em um tema complexo, atingindo de forma positiva no aprendizado dos alunos e, até mesmo, no ensino dos docentes da área.

Palavras-chave: Web Software, Engenharia Civil, Lajes, Educação, Concreto Armado.

\section{DEVELOPMENT OF EDUCATIONAL SOFTWARE AS A TEACHING TOOL IN CIVIL ENGINEERING - SIMPLE REINFORCED CONCRETE SLABS}

\begin{abstract}
This project deals with the development of software capable of designing simple slabs, through the processing of data in a web application. It was created due to the need of civil engineering students for a good way to study and correct their exercises, which is proposed in this program. With the demonstration, through the resolution of a typical exercise, it was possible to observe the effectiveness with which the program exercises its function in a complex theme, reaching in a positive way in the student's learning and, even, in the tutoring of the teachers of the area.
\end{abstract}

Keywords: Web Software, Civil Engineering, Slabs, Education, Reinforced Concrete. 


\section{INTRODUÇÃO}

O presente relatório é fruto do trabalho desenvolvido durante o período de Iniciação Científica, disponível pelo edital PIBIC 2018, como aluno sem bolsa, do curso de engenharia civil da instituição de ensino CEFET/RJ. Este trabalho surgiu de uma necessidade dos alunos de engenharia civil, de uma forma prática e rápida, estudar e resolver problemas com o auxílio de programas computacionais. Recentemente, com o avanço da tecnologia e da maneabilidade das linguagens de programação, unidos ao conhecimento técnico da engenharia, foi possível o surgimento de softwares capazes de realizar complexos cálculos e modelagens, de forma a entregar certa praticidade, tanto para o aluno, que o utiliza para estudos acadêmicos, tanto para o futuro profissional da área, atuando em projetos do cotidiano, garantindo, à ambos, agilidade e segurança em seus respectivos objetivos.

Por meio de experiência própria do autor e da influência de softwares já existentes que auxiliam no campo da engenharia, principalmente de estruturas, como o Ftool, desenvolvido pela PUC-Rio, observou-se a facilidade atingida pela parte docente e discente no ensino de determinadas disciplinas, pela rapidez, clareza e integração dos exercícios com a matéria lecionada, tornando-o praticamente essencial para o completo aprendizado da cadeira. Com tal incentivo, foi concebido este projeto, visando, por meio de um software de dimensionamento de lajes maciças simples em concreto armado, obter resultado semelhante.

\section{METODOLOGIA}

Tratasse da metodologia utilizada para o cálculo de lajes maciças simples de concreto armado e para o desenvolvimento do software elaborado.

\subsection{Dimensionamento da laje maciça simples}

O dimensionamento de uma laje maciça simples é feito conforme a distribuição de sua armadura, o qual pode ser feita em uma ou duas direções. De acordo com Bastos (2015), as lajes armadas em uma direção são calculadas como vigas segundo a direção principal e as lajes armadas em duas direções podem ser aplicadas diferentes teorias. Dentre estas, foi selecionada a teoria das grelhas, por ser um método que, segundo Araújo (2010) pode ser facilmente utilizado para elaborar um programa de computador para o cálculo de lajes retangulares. Além disso, desta 
teoria se baseia o método de Marcus, que é um método simplificado que procura adaptar a teoria das grelhas para incluir os efeitos de torção da laje (ARAÚJO,2010).

Tal método foi escolhido por ser vastamente lecionado nas instituições de ensino de engenharia e por apresentar uma forma menos complexa e mais intuitiva de calcular os momentos fletores solicitantes nas lajes, por meio do uso de tabelas.

\subsection{Confecção do software}

Para a criação do software resultante deste trabalho, foi necessário, primeiramente, a determinação do tipo de programa a ser elaborado. Assim, dentre as diversas formas de elaborar um programa, o formato de aplicação web foi o escolhido, por diversas vantagens: a praticidade de utilização, por não necessitar de instalação prévia; o largo alcance de usuários, possibilitando o seu uso desde computadores, até mesmo smartphones e outros aparelhos mobile.Com isso, foi necessário o aprendizado nas linguagens básicas para elaboração de conteúdo web: HTML, CSS e JavaScript; para, basicamente, a formatação de páginas, estilização de conteúdo e programação front-end, respectivamente. No entanto, esta última foi substituída pela linguagem PHP, a fim de se obter a vantagem do processamento em servidores, melhorando a eficiência e abrangendo ainda mais o alcance de usuários, por retirar a necessidade de especificações técnicas mínimas por parte do usuário. Além dessas linguagens, também foi praticado o paradigma de Programação Orientada ao Objeto (POO), que se apresenta muito prático para a prototipagem e organização do programa, principalmente no que tange à sua manutenção e atualização. Com o software definido, iniciou-se a montagem do código, com 3 diferentes classes: uma "classe-mãe", e duas "classes-filhas", tratando, respectivamente, das características gerais da laje e das características específicas da armação utilizada na mesma. Além disso, foi determinado um algoritmo de execução do programa, que prossegue desde a entrada de dados da estrutura; a escolha do tipo de caso, entre uma laje bidirecional ou unidirecional; a obtenção de valores relacionados aos momentos e reações verticais nos apoios, até a apresentação dos resultados ao usuário.

\section{RESULTADOS}

Nesta parte do artigo será apresentado o software desenvolvido, junto à exercício para demonstração do funcionamento do mesmo. 


\subsection{O software}

O software é composto por duas páginas distintas, sendo a primeira, representada na Erro! Fonte de referência não encontrada.Erro! Fonte de referência não encontrada., referente as entradas de dados no programa, onde serão inseridos as informações referentes à laje a ser calculada: as condições do contorno da laje, as medidas nas direções $\mathrm{X}$ e $\mathrm{Y}$, a carga de projeto, a espessura, o cobrimento, o fck do concreto e o tipo de aço que vai ser utilizado. Já na segunda página, estão registrados os resultados dos momentos fletores solicitantes e das reações nos apoios, de acordo com as informações de entrada, além do cálculo das áreas de armaduras necessárias, assim como possíveis soluções de distribuição das armaduras principais ao longo da estrutura.

O software se encontra na plataforma virtual do projeto, denominada Civil Engineering Learning Tools (CELT), onde estão dispostos demais ferramentas desenvolvidas por outros alunos, através do projeto de extensão "Programação WEB para Desenvolvimento de Software Educacional como Ferramenta de Ensino para a Engenharia Civil"

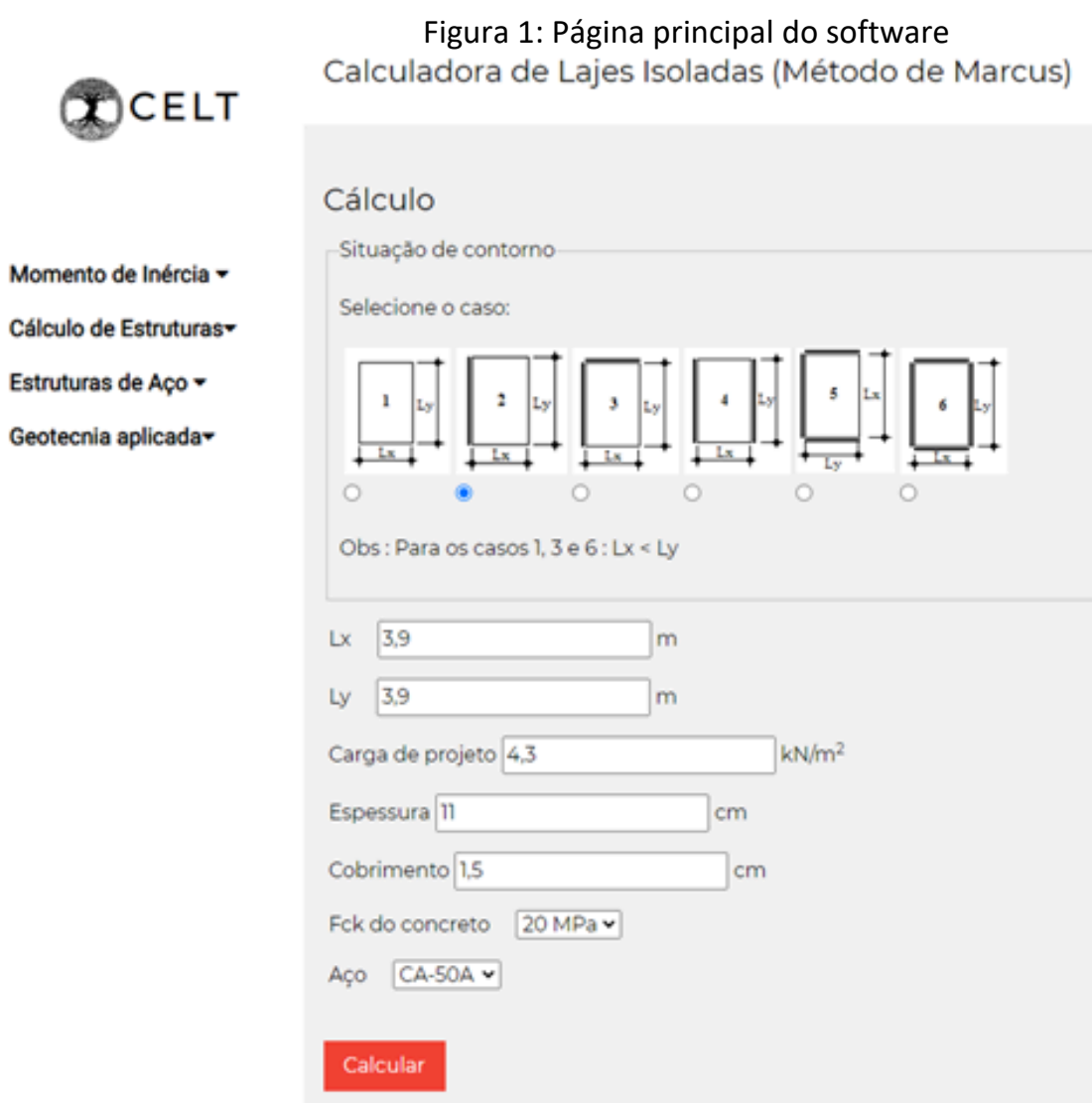

Fonte: (Autor, 2021) 


\subsection{Apresentação de problema típico}

A Figura 2 apresenta um exemplo de exercício comumente apresentado em sala de aula, obtido de BOTELHO (2002). Para esta laje, pede-se os momentos fletores solicitados na estrutura para duas situações de carga (q) diferentes: uma carga comum, de 4,3 kN/m², denominada de carga 1; e uma sobrecarga industrial de $12,8 \mathrm{kN} / \mathrm{m}^{2}$, denominada de carga 2 . Foi adotada a espessura de $11 \mathrm{~cm}$, com 1,5 cm de cobrimento da armadura, aço - 50A e fck de $15 \mathrm{MPa}$ para o concreto.

Figura 2: Exercício para cálculo de laje

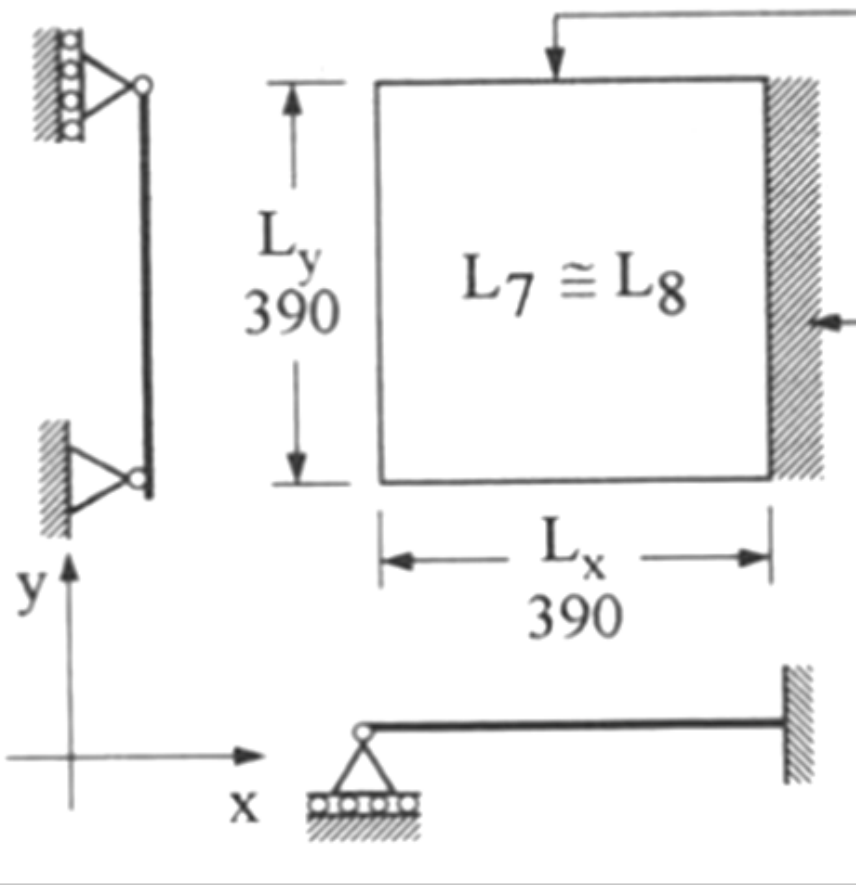

Fonte: (BOTELHO,2002).

\subsection{Resolução do livro}

Para a resolução do problema, a primeira etapa é verificar se a laje é unidirecional ou bidirecional, por meio da razão $(\lambda)$ entre as dimensões na direção y e na direção x: caso essa razão apresente valor maior que 2 , esta laje será classificada como unidirecional, tendo seus momentos calculados como uma viga ao longo da menor direção; caso essa razão seja menor ou igual a 2, a mesma será calculada, neste caso, pelo método de Marcus. Como a laje apresenta medidas iguais em ambas as direções, sua razão possui o valor de 1, sendo assim determinada como uma laje armada em cruz. 
Para o seu cálculo pelo método de Marcus, primeiramente é necessário classificar o caso em que a laje está classificada. Na Figura 3 está demonstrado os seis diferentes casos possíveis de comportamento da laje com relação a sua condição de contorno. Esta condição é ditada pela situação em que os bordos da laje se encontram, considerando dois casos principais: o apoio simplesmente apoiado, onde não há a ocorrência de momentos fletores neste apoio, por possuir maior liberdade; e o apoio engastado, onde há a ocorrência de momentos no apoio, devido ao engaste da peça.

Figura 3: Representação dos casos da laje
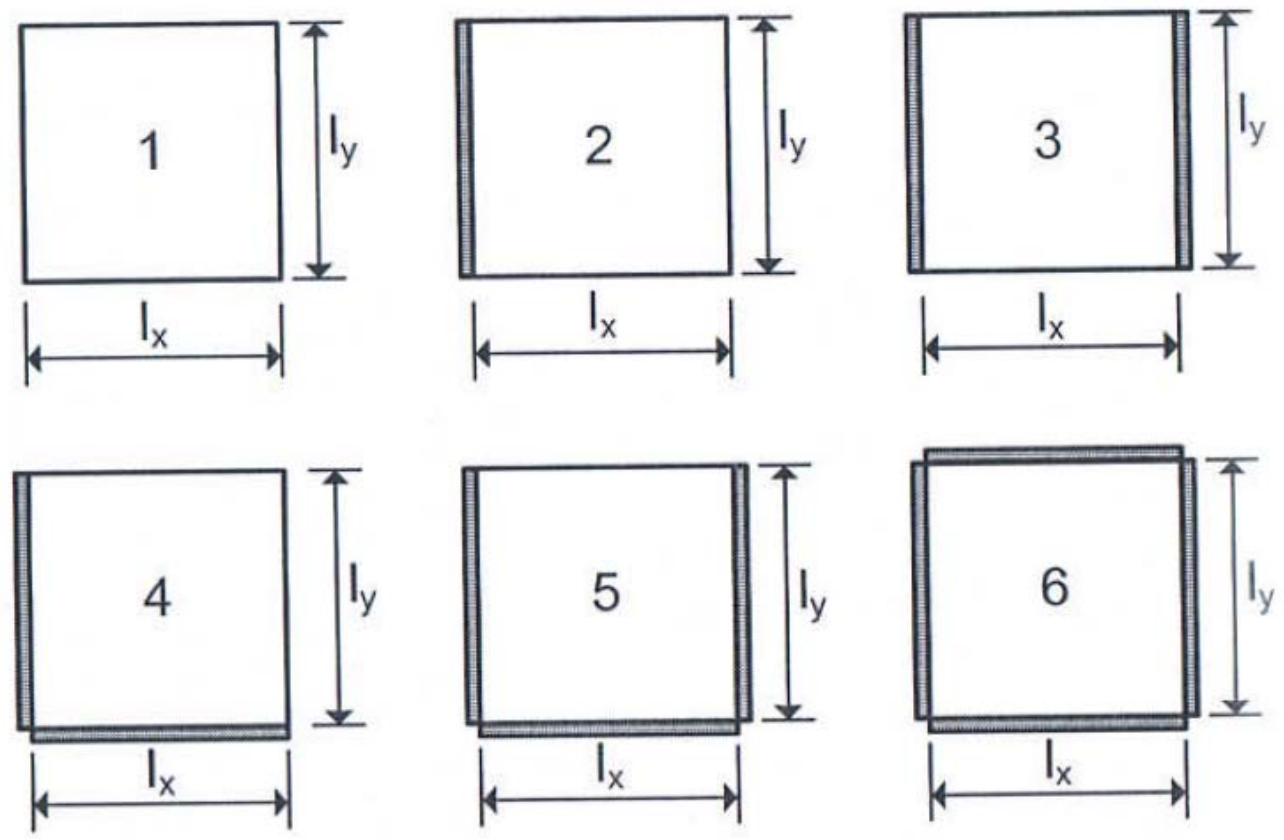

Fonte: (ARAÚJO,2010).

Já na Figura 4, apresenta-se uma das tabelas utilizadas para o dimensionamento da laje, para o caso apresentado no exercício, junto aos coeficientes utilizados pelo método e as fórmulas para o cálculo dos diferentes momentos possíveis: no centro da laje na direção x (Mx); na direção y (My); e dos momentos nos apoios engastados na direção x (Mex); e na direção y (Mey). 
Figura 4: Coeficientes e fórmulas para o caso 2, pelo método de Marcus

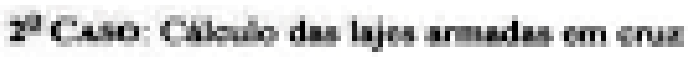

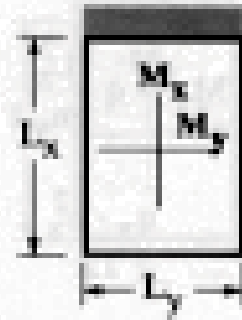

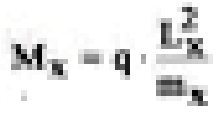

$$
\begin{aligned}
& \mathbf{M}_{y}=\mathbf{q}-\frac{L_{x}^{2}}{\mathbf{m}_{y}} \\
& 4 x=k_{x}-4 \quad 4 y-4=4 x \\
& x_{x}=-\frac{4 \cdot-12}{n}
\end{aligned}
$$

\begin{tabular}{|c|c|c|c|c|c|c|c|c|c|c|c|}
\hline$L_{1}$ & $\mathbf{m i}_{\mathbf{i}}$ & 17, & $\mathbf{L}_{\mathbf{L}}$ & $\mathrm{L}_{\mathrm{x}}$ & $m_{i}$ & $\mathbf{m}_{z}$ & $E_{4}$ & $L_{1} L_{L}$ & $\theta_{4}$ & $\mathrm{~m}_{\mathrm{t}}$ & $\mathbf{E}_{\mathbf{E}}$ \\
\hline 0,40 & 140,9 & 45,1 & 9,124 & $1, \mathbf{6 0}$ & 24,9 & 16,7 & 0,714 & 1,40 & 140 & 96,7 & 1925 \\
\hline 0,5 & & & & & & & & & & & \\
\hline 054 & & & & & & & & & & & \\
\hline 94 & & & & & & & & & & & \\
\hline as & & & & (t) & & & & & & & \\
\hline & & & & : & & & & & & & \\
\hline 0 & & & & & & & & & & & \\
\hline 0 & & & & & & & & & & & \\
\hline 0 & & & & & & & & & & & \\
\hline 0 & & & & & & & & & & & \\
\hline 0.5 & & & & & & & & & & & \\
\hline 0,7 & & & & & & & & & & & \\
\hline 0,7 & & & & & & & & & & & \\
\hline 0,7 & & & & & & & & & & & \\
\hline 0,7 & & & & & & & & & & & \\
\hline $0, \mathbb{B}$ & & & & & & & & & & & \\
\hline 0,62 & & & & & & & & & & & \\
\hline & & & & & & & & & & & \\
\hline 0,86 & & & & & & & & & & & \\
\hline 9.kh & & & & & & 40,4 & & & & & $4 t$ \\
\hline 900 & & & & & & & & & & & 970 \\
\hline 0,42 & & & & & & & & & & 9.2 & \\
\hline 0,04 & & & & 1,44 & & 33,2 & & & 16,5 & 4,7 & 97 \\
\hline & & & & & & & & & & 6,2 & \\
\hline & & & & & & & & & & 17,7 & \\
\hline 1,00 & 39 & 367 & 0714 & 1,50 & 19,0 & 53 & 0,926 & 2,0 & 16,5 & $m_{1}=$ & 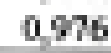 \\
\hline
\end{tabular}

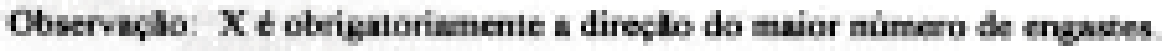

Fonte: (BOTELHO,2002).

Por possuir apoios simples em todas as bordas, com exceção da borda lateral direita, que está engastada, esta laje está classificada no 2 o caso desta classificação. Assim, é possível obter o resultado dos momentos fletores solicitados, para ambas as cargas. Quanto ao dimensionamento da armadura principal, são utilizadas, da mesma forma, tabelas para a obtenção da área de armadura por metro necessária (As) e do diâmetro do aço a ser utilizado, com o seu espaçamento. 
Por meio da Equação 1 (BOTELHO,2008), é possível obter o valor de k6, que, por sua vez, é inserido na Tabela 1, de forma a encontrar o coeficiente $k 3$.

$$
k_{6}=\frac{10^{5} \cdot b \cdot d^{2}}{M}
$$

Onde:

b: $100 \mathrm{~cm}$ (cálculo por metro);

d: Distancia da borda mais comprimida ao centro de gravidade da armadura (m);

$\mathrm{M}$ : Momento fletor na peça $(\mathrm{kNm})$.

Tabela 1: Tabela para obtenção do coeficiente k3

\begin{tabular}{|c|c|c|c|c|c|c|c|c|c|c|c|}
\hline \multicolumn{3}{|c|}{$\begin{array}{c}\text { Valores de k6 para } \\
\text { concreto }\end{array}$} & \multicolumn{3}{|c|}{ k3/aço } & \multicolumn{3}{|c|}{$\begin{array}{l}\text { Valores de k6 para } \\
\text { concreto }\end{array}$} & \multicolumn{3}{|c|}{ k3/aço } \\
\hline \multicolumn{3}{|c|}{ fck (MPa) } & \multicolumn{3}{|c|}{$\mathrm{CA}$} & \multicolumn{3}{|c|}{ fck (MPa) } & \multicolumn{3}{|c|}{ CA } \\
\hline 20 & 25 & 30 & 25 & $50 \mathrm{~A}$ & $60 \mathrm{~B}$ & 20 & 25 & 30 & 25 & $50 \mathrm{~A}$ & $60 \mathrm{~B}$ \\
\hline 1447 & 1158 & 965 & 0,647 & 0,323 & 0,269 & 59,8 & 47,9 & 39,9 & 0,722 & 0,361 & 0,301 \\
\hline 726 & 581 & 484 & 0,649 & 0,325 & 0,271 & 58 & 46,4 & 38,6 & 0,725 & 0,363 & 0,302 \\
\hline 486 & 389 & 324 & 0,652 & 0,326 & 0,272 & 56,2 & 45 & 37,5 & 0,729 & 0,364 & 0,304 \\
\hline 366 & 293 & 244 & 0,655 & 0,327 & 0,273 & 54,6 & 43,7 & 36,4 & 0,732 & 0,366 & 0,305 \\
\hline 294 & 235 & 196 & 0,657 & 0,329 & 0,274 & 53,1 & 42,5 & 35,4 & 0,735 & 0,368 & 0,306 \\
\hline 246 & 197 & 164 & 0,66 & 0,33 & 0,275 & 51,6 & 41,3 & 34,4 & 0,739 & 0,369 & 0,308 \\
\hline 212 & 169 & 141 & 0,663 & 0,331 & 0,276 & 50,3 & 40,3 & 33,5 & 0,742 & 0,371 & 0,309 \\
\hline 186 & 149 & 124 & 0,665 & 0,333 & 0,277 & 49,1 & 39,2 & 32,7 & 0,746 & 0,373 & 0,311 \\
\hline 166 & 133 & 111 & 0,668 & 0,334 & 0,278 & 47,9 & 38,3 & 31,9 & 0,749 & 0,374 & 0,312 \\
\hline 150 & 120 & 100,1 & 0,671 & 0,335 & 0,28 & 46,8 & 37,4 & 31,2 & 0,752 & 0,376 & 0,313 \\
\hline 137 & 110 & 91,4 & 0,674 & 0,337 & 0,281 & 45,7 & 36,6 & 30,5 & 0,756 & 0,378 & 0,315 \\
\hline 126 & 100,9 & 84,1 & 0,677 & 0,338 & 0,282 & 44,7 & 35,8 & 29,8 & 0,76 & 0,38 & 0,316 \\
\hline 117 & 93,6 & 78 & 0,679 & 0,34 & 0,283 & 43,8 & 35 & 29,2 & 0,763 & 0,382 & 0,318 \\
\hline 109 & 87,2 & 72,7 & 0,682 & 0,341 & 0,284 & 42,9 & 34,3 & 28,6 & 0,767 & 0,383 & 0,319 \\
\hline 102,2 & 81,8 & 68,1 & 0,685 & 0,343 & 0,285 & 42 & 33,6 & 28 & 0,77 & 0,385 & 0,321 \\
\hline 96,2 & 77 & 64,2 & 0,688 & 0,344 & 0,287 & 41,2 & 33 & 27,5 & 0,774 & 0,387 & 0,323 \\
\hline
\end{tabular}

Fonte: (BOTELHO,2008).

Tabela 1: Tabela para obtenção do coeficiente k3 - Continuação

\begin{tabular}{c|c|c|c|c|c|c|c|c|c|c|c}
\hline \multicolumn{2}{c|}{$\begin{array}{c}\text { Valores de k6 para } \\
\text { concreto }\end{array}$} & \multicolumn{3}{c|}{ k3/aço } & \multicolumn{3}{c|}{$\begin{array}{c}\text { Valores de k6 para } \\
\text { concreto }\end{array}$} & \multicolumn{3}{c}{ k3/aço } \\
\hline \multicolumn{3}{c|}{ fck (MPa) } & \multicolumn{3}{c|}{ CA } & \multicolumn{3}{c|}{ fck (MPa) } & \multicolumn{3}{c}{ CA } \\
\hline $\mathbf{2 0}$ & 25 & 30 & 25 & $50 A$ & $60 B$ & 20 & 25 & 30 & 25 & $50 A$ & $60 B$ \\
\hline $\mathbf{9 1}$ & 72,8 & 60,6 & 0,691 & 0,346 & 0,288 & 40,5 & 32,4 & 27 & 0,778 & 0,389 & 0,324 \\
\hline $\mathbf{8 6 , 3}$ & 69 & 57,5 & 0,694 & 0,347 & 0,289 & 39 & 31,8 & 26,5 & 0,782 & 0,391 & 0,326 \\
\hline $\mathbf{8 2 , 1}$ & 65,7 & 54,7 & 0,697 & 0,349 & 0,29 & 39,1 & 31,2 & 26 & 0,786 & 0,393 & - \\
\hline $\mathbf{7 8 , 3}$ & 62,7 & 52,2 & 0,7 & 0,35 & 0,292 & 38,4 & 30,7 & 25,6 & 0,789 & 0,395 & - \\
\hline $\mathbf{7 4 , 9}$ & 59,9 & 49,9 & 0,703 & 0,352 & 0,293 & 37,8 & 30,2 & 25,2 & 0,793 & 0,397 & - \\
\hline $\mathbf{7 1 , 8}$ & 57,5 & 47,9 & 0,706 & 0,353 & 0,294 & 37,2 & 29,7 & 24,8 & 0,797 & 0,399 & - \\
\hline $\mathbf{6 9}$ & 55,2 & 46 & 0,709 & 0,355 & 0,296 & 36,6 & 29,3 & 24,4 & 0,801 & 0,401 & - \\
\hline $\mathbf{6 4 , 1}$ & 51,2 & 42,7 & 0,716 & 0,358 & 0,298 & 36 & 28,8 & 24 & 0,805 & 0,403 & - \\
\hline $\mathbf{6 1 , 9}$ & 49,5 & 41,2 & 0,719 & 0,359 & 0,3 & - & - & - & - & - & - \\
\hline
\end{tabular}

Fonte: (BOTELHO,2008). 
Com a obtenção do valor de k3, por meio da Equação 2 (BOTELHO,2008), é possível obter o valor de As. A partir deste valor, consulta-se a Tabela 2, de forma a obter as soluções possíveis para o diâmetro e distribuição da armadura ao longo da estrutura. Ambos os coeficientes estão relacionados ao fck do concreto e ao tipo de aço utilizado na armadura, onde, o uso de diferentes tipos de resistências para o concreto e diferentes tipos de aço, para a mesma situação, resultarão em diversas soluções possíveis, onde a versatilidade de um programa computacional torna-se conveniente.

$$
A_{s}=\frac{k_{3}}{10} \times \frac{M}{d}
$$

Onde:

As: Área de armadura da seção $\left(\mathrm{cm}^{2} / \mathrm{m}\right)$;

d: Distancia da borda mais comprimida ao centro de gravidade da armadura (m);

$\mathrm{M}$ : Momento fletor na peça $(\mathrm{kNm})$.

Tabela 2: Diâmetro e espaçamento do aço com relação à área de armadura por metro

\begin{tabular}{|c|c|c|c|c|c|c|}
\hline \multicolumn{7}{|c|}{ Área em $\mathrm{cm}^{2} / \mathrm{m}$ de armadura } \\
\hline Espaçamento & \multicolumn{6}{|c|}{ Diâmetro do aço } \\
\hline- & 5 & 6,3 & 8 & 10 & 12,5 & 16 \\
\hline 7,5 & 3,33 & 4,19 & 6,66 & 10,66 & 16,66 & 26,66 \\
\hline 8 & 2,5 & 3,93 & 6,25 & 10 & 15,62 & 25 \\
\hline 9 & 2,22 & 3,5 & 5,55 & 8,88 & 13,88 & 22,22 \\
\hline 10 & 2 & 3,15 & 5 & 8 & 12,5 & 20 \\
\hline 11 & 1,82 & 2,86 & 4,54 & 7,27 & 11,36 & 18,18 \\
\hline 12 & 1,67 & 2,62 & 4,16 & 6,66 & 10,41 & 16,66 \\
\hline 12,5 & 1,6 & 2,52 & 4 & 6,4 & 10 & 16 \\
\hline 13 & 1,54 & 2,42 & 3,84 & 6,15 & 9,61 & 15,38 \\
\hline 14 & 1,43 & 2,25 & 3,57 & 5,71 & 8,92 & 14,28 \\
\hline 15 & 1,33 & 2,1 & 3,33 & 5,33 & 8,33 & 13,33 \\
\hline 16 & 1,25 & 1,96 & 3,12 & 5 & 7,81 & 12,5 \\
\hline 17 & 1,18 & 1,85 & 2,94 & 4,7 & 7,35 & 11,76 \\
\hline 18 & 1,11 & 1,75 & 2,77 & 4,44 & 6,94 & 11,11 \\
\hline 19 & 1,05 & 1,65 & 2,63 & 4,21 & 6,57 & 10,52 \\
\hline 20 & 1 & 1,57 & 2,5 & 4 & 6,25 & 10 \\
\hline 21 & 0,95 & 1,5 & 2,38 & 3,8 & 5,95 & 9,52 \\
\hline 22 & 0,91 & 1,43 & 2,27 & 3,63 & 5,68 & 9,09 \\
\hline 23 & 0,87 & 1,36 & 2,17 & 3,47 & 5,43 & 8,69 \\
\hline 24 & 0,83 & 1,31 & 2,08 & 3,33 & 5,2 & 8,33 \\
\hline 25 & 0,8 & 1,26 & 2 & 3,2 & 5 & 8 \\
\hline 26 & 0,77 & 1,21 & 1,92 & 3,07 & 4,8 & 7,69 \\
\hline 27 & 0,74 & 1,16 & 1,85 & 2,96 & 4,62 & 7,4 \\
\hline 28 & 0,71 & 1,12 & 1,78 & 2,85 & 4,46 & 7,14 \\
\hline 29 & 0,69 & 1,08 & 1,72 & 2,75 & 4,31 & 6,89 \\
\hline 30 & 0,67 & 1,05 & 1,66 & 2,66 & 4,16 & 6,66 \\
\hline
\end{tabular}

Fonte: (BOTELHO,2008). 
Assim, são obtidos os resultados dos momentos e as áreas de armadura das cargas 1 e 2, dispostos, respectivamente, nas Tabelas 3 e 4.

Tabela 3: Dimensionamento da laje submetida a carga 1

\begin{tabular}{c|c|c|c}
\hline & Direção $X$ & Direção $Y$ & Engaste em $X$ \\
\hline Momentos $(\mathrm{kNm})$ & 2,20 & 1,80 & $-5,90$ \\
\hline As $\left(\mathrm{cm}^{2} / \mathrm{m}\right)$ & 0,76 & 0,62 & 2,11 \\
\hline Armadura principal & $\begin{array}{c}\varnothing 6,3 \mathrm{~mm} \text { a cada } 20 \\
\mathrm{~cm}\end{array}$ & $\varnothing 6,3 \mathrm{~mm}$ a cada $20 \mathrm{~cm}$ & $\varnothing 6,3 \mathrm{~mm}$ a cada $15 \mathrm{~cm}$ \\
\hline \multicolumn{2}{|c}{ Fonte: (BOTELHO,2002). }
\end{tabular}

Tabela 4: Dimensionamento da laje submetida a carga 2

\begin{tabular}{c|c|c|c}
\hline & Direção $X$ & Direção $\mathrm{Y}$ & Engaste em $\mathrm{X}$ \\
\hline Momentos $(\mathrm{kNm})$ & 6,40 & 5,30 & $-17,4$ \\
\hline As $\left(\mathrm{cm}^{2} / \mathrm{m}\right)$ & 2,34 & 1,89 & 7,22 \\
\hline Armadura principal & $\varnothing 8 \mathrm{~mm}$ a cada $20 \mathrm{~cm}$ & $\varnothing 8 \mathrm{~mm}$ a cada $20 \mathrm{~cm}$ & $\varnothing 12,5 \mathrm{~mm}$ a cada $17,5 \mathrm{~cm}$ \\
\hline
\end{tabular}

Fonte: (BOTELHO,2002).

3.4. Resolução computacional por meio do software

O software, diferentemente do método de consulta de tabelas, para o método de Marcus, ele utiliza diretamente de equações do método de teoria das grelhas para lajes sobre apoios rígidos para obtenção dos parâmetros tabelados, em cada caso, demonstrados no Quadro 1. Obtidos os coeficientes kx e ky, inicialmente, obtêm-se os coeficientes para o cálculo dos momentos fletores positivos no centro da laje da direção $x(m x)$ e na direção y (my); e dos momentos negativos presentes nos engastes, na direção $x$ (mex) e na direção y (mey), onde calcula-se o momento com uso da Equação 3 (ARAÚJO,2010).

$$
M=m p l_{x}^{2}
$$

Onde:

$\mathrm{M}$ : Momento calculado para determinado ponto $(\mathrm{kNm})$;

p: Carga de projeto $\left(\mathrm{kN} / \mathrm{m}^{2}\right)$;

$\mathrm{I}_{\mathrm{x}}$ : Vão efetivo na direção $\mathrm{x}(\mathrm{m})$. 
Quadro 1: Formulário do método de teoria das grelhas para lajes sobre apoios rígidos para cada caso

\begin{tabular}{c|c|c|c|c|c|c}
\hline & Caso 1 & Caso 2 & Caso 3 & Caso 4 & Caso 5 & Caso 6 \\
\hline $\mathrm{kx}$ & $\frac{\lambda^{4}}{1+\lambda^{4}}$ & $\frac{5 \lambda^{4}}{2+5 \lambda^{4}}$ & $\frac{5 \lambda^{4}}{1+5 \lambda^{4}}$ & $\frac{\lambda^{4}}{1+\lambda^{4}}$ & $\frac{2 \lambda^{4}}{1+2 \lambda^{4}}$ & $\frac{\lambda^{4}}{1+\lambda^{4}}$ \\
\hline $\mathrm{ky}$ & $1-k_{x}$ & $1-k_{x}$ & $1-k_{x}$ & $1-k_{x}$ & $1-k_{x}$ & $1-k_{x}$ \\
\hline $\mathrm{my}$ & $\frac{k_{x}}{8}$ & $\frac{k_{x}}{14,22}$ & $\frac{k_{x}}{24}$ & $\frac{k_{x}}{14,22}$ & $\frac{k_{x}}{24}$ & $\frac{k_{x}}{24}$ \\
\hline $\mathrm{mex}$ & $\frac{k_{y} \lambda^{2}}{8}$ & $\frac{k_{y} \lambda^{2}}{8}$ & $\frac{k_{y} \lambda^{2}}{8}$ & $\frac{k_{y} \lambda^{2}}{14,22}$ & $\frac{k_{y} \lambda^{2}}{14,22}$ & $\frac{k_{y} \lambda^{2}}{24}$ \\
\hline $\mathrm{mey}$ & - & $-\frac{k_{x}}{8}$ & $-\frac{k_{x}}{12}$ & $-\frac{k_{x}}{8}$ & $-\frac{k_{x}}{12}$ & $-\frac{k_{x}}{12}$ \\
\hline
\end{tabular}

Fonte:(ARAÚJO,2010)

Com estes coeficientes, é possível calcular o momento fletor desejado na peça estrutural. Os coeficientes Cx e Cy são calculados por meio das Equações 4 e 5 (ARAÚJO,2010), que, por sua vez, serão multiplicados aos valores dos momentos obtidos pelo método das grelhas, de forma a obter o momento fletor final, pelo método de Marcus.

$$
\begin{aligned}
& C_{x}=1-\frac{20 k_{x}}{3 \alpha_{x} \lambda^{2}} \\
& C_{y}=1-\frac{20 k_{y} \lambda^{2}}{3 \alpha_{y}}
\end{aligned}
$$

Onde:

$\alpha=8$, para uma faixa biapoiada;

$\alpha=14,22$, para uma faixa engastada e apoiada;

$\alpha=24$, para uma faixa biengastada.

Para o cálculo das reações de apoio, foram utilizadas as expressões presentes na Figura 5, em conjunto com a representação gráfica das reações, utilizando o processo das áreas de influência, para os casos 1 a 6 do método de Marcus. 
Figura 5: Formulário para o cálculo das reações de apoio pelo processo das áreas de influência

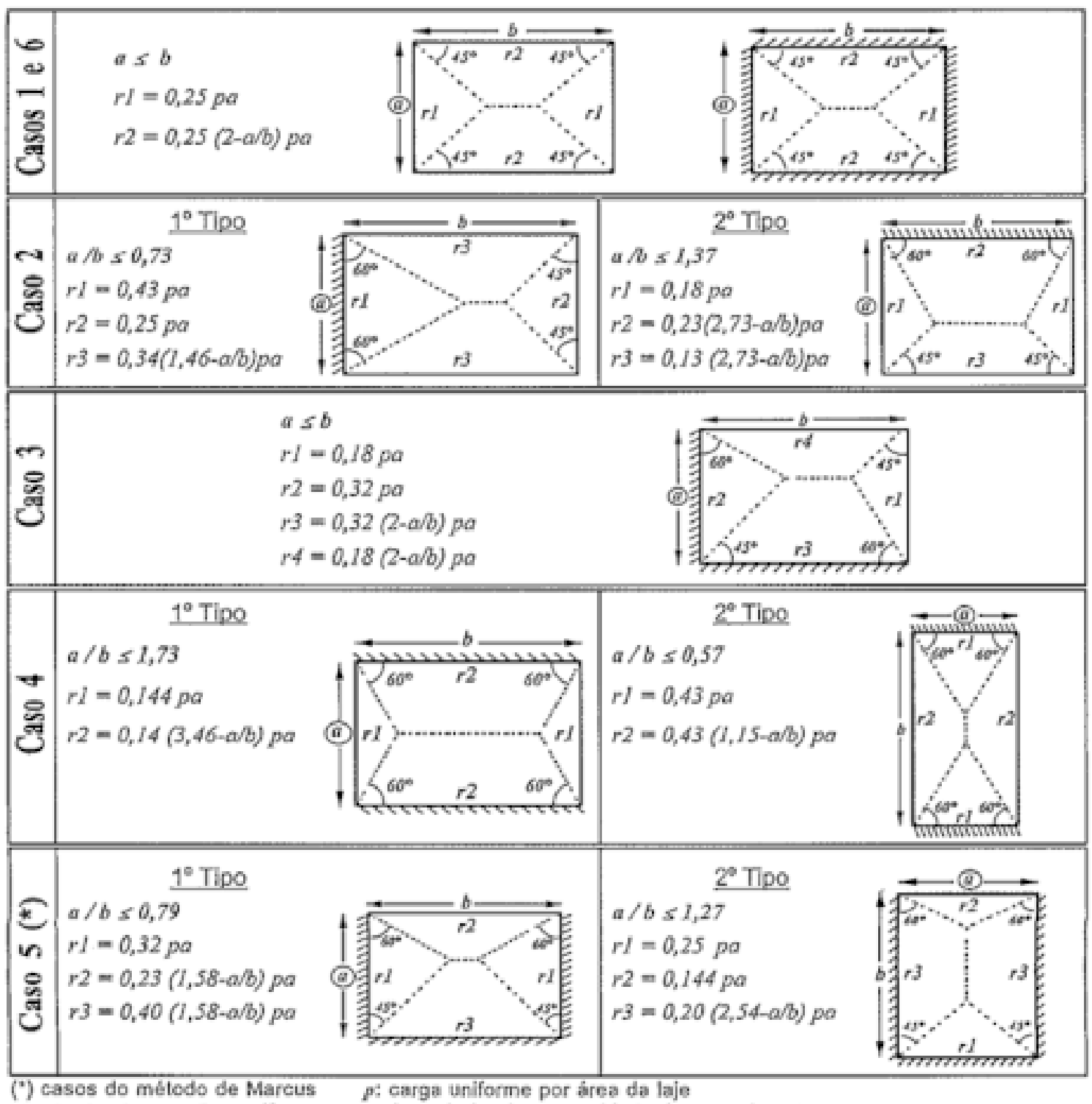

$r 1, r 2, r 3, r 4=$ reaçסes uniformes nas vigas de bordo, por unidade de comprimento

Fonte: (CLÍMACO,2008)

Para o dimensionamento da armadura, a aplicação segue, igualmente, o processo de cálculo por tabelas. Além disso, para o cálculo da taxa de armadura, é feito uma verificação pelo software, de forma a obedecer ao item 17.3.5 da NBR 6118 (ABNT,2014), onde a mesma não deve ser menor que $0,15 \%$ da área de aço dividida pela área de concreto, para a resistência do concreto utilizada no programa. Desta forma, para o problema em questão, foi exemplificado uma tela de entrada de dados, para a carga 1, apresentada na Figura 1 e relatou-se, pelo software, a página presente nas Figuras 6 e 7, referentes a mesma carga. Os demais resultados, dos momentos fletores e dimensionamento de armadura, estão apresentados nas Tabelas 5 e 6. 
Figura 6: Cálculo dos momentos e reações nos apoios da laje pelo software para a carga 1

\section{(2) CELT}

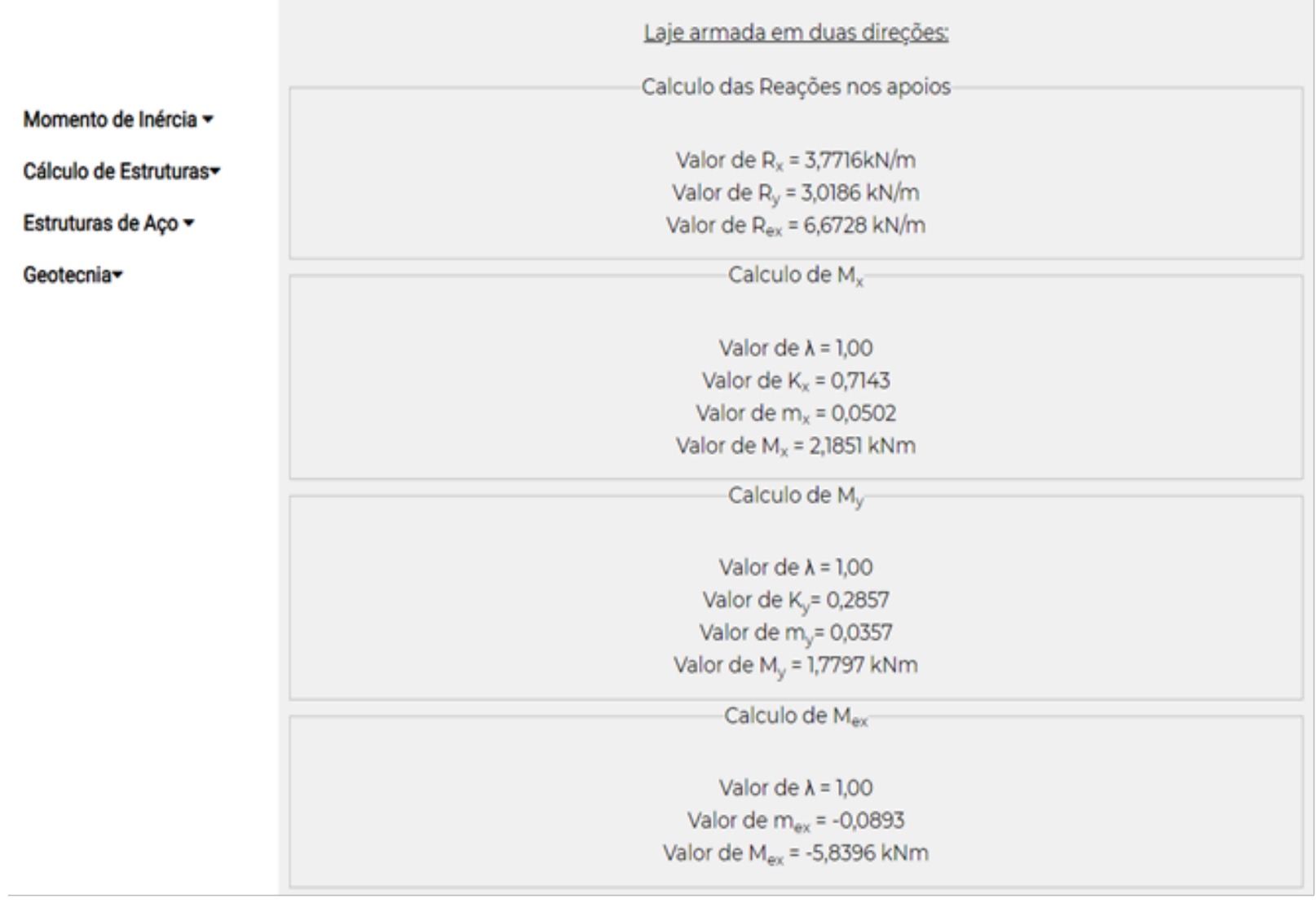

Fonte: (Autor, 2021)

Tabela 5: Dimensionamento da estrutura submetida a carga 1 pelo software

\begin{tabular}{c|c|c|c}
\hline & Direção X & Direção $Y$ & Engaste em X \\
\hline Momentos $(\mathrm{kNm})$ & 2,1851 & 1,7797 & $-5,8396$ \\
\hline As $\left(\mathrm{cm}^{2} / \mathrm{m}\right)$ & 0,75 & 0,61 & 2,06 \\
\hline As adotada $\left(\mathrm{cm}^{2} / \mathrm{m}\right)$ & 1,65 & 1,65 & 2,06 \\
\hline $\begin{array}{c}\text { Armadura } \\
\text { principal }\end{array}$ & $\varnothing 5 \mathrm{~mm}$ a cada $12 \mathrm{~cm}$. & $\varnothing 5 \mathrm{~mm}$ a cada $12 \mathrm{~cm}$. & $\varnothing 5 \mathrm{~mm}$ a cada $9 \mathrm{~cm}$. \\
\hline
\end{tabular}

Fonte: (Autor, 2021)

\section{Resultado:}

\section{Calculo dos Momentos e Reações nos apoios}

\section{Laje armada em duas direçōes:}

Valor de $\mathrm{R}_{\mathrm{x}}=3,7716 \mathrm{kN} / \mathrm{m}$

Valor de $R_{y}=3,0186 \mathrm{kN} / \mathrm{m}$

Calculo de $M_{x}$

Valor de $\lambda=1,00$

valor de $K_{x}=0,7143$

Valor de $m_{x}=0,0502$

Valor de $\mathrm{A}=1,00$

Valor de $K_{y}=0,2857$

valor de $m_{\mathrm{y}}=0,0357$

Valor de $\lambda=1,00$

Valor de $m_{\text {ex }}=-0,0893$

valor de $M_{\text {ex }}=-5,8396 \mathrm{kNm}$ 
Figura 7: Dimensionamento da armadura da laje pelo software para a carga 1

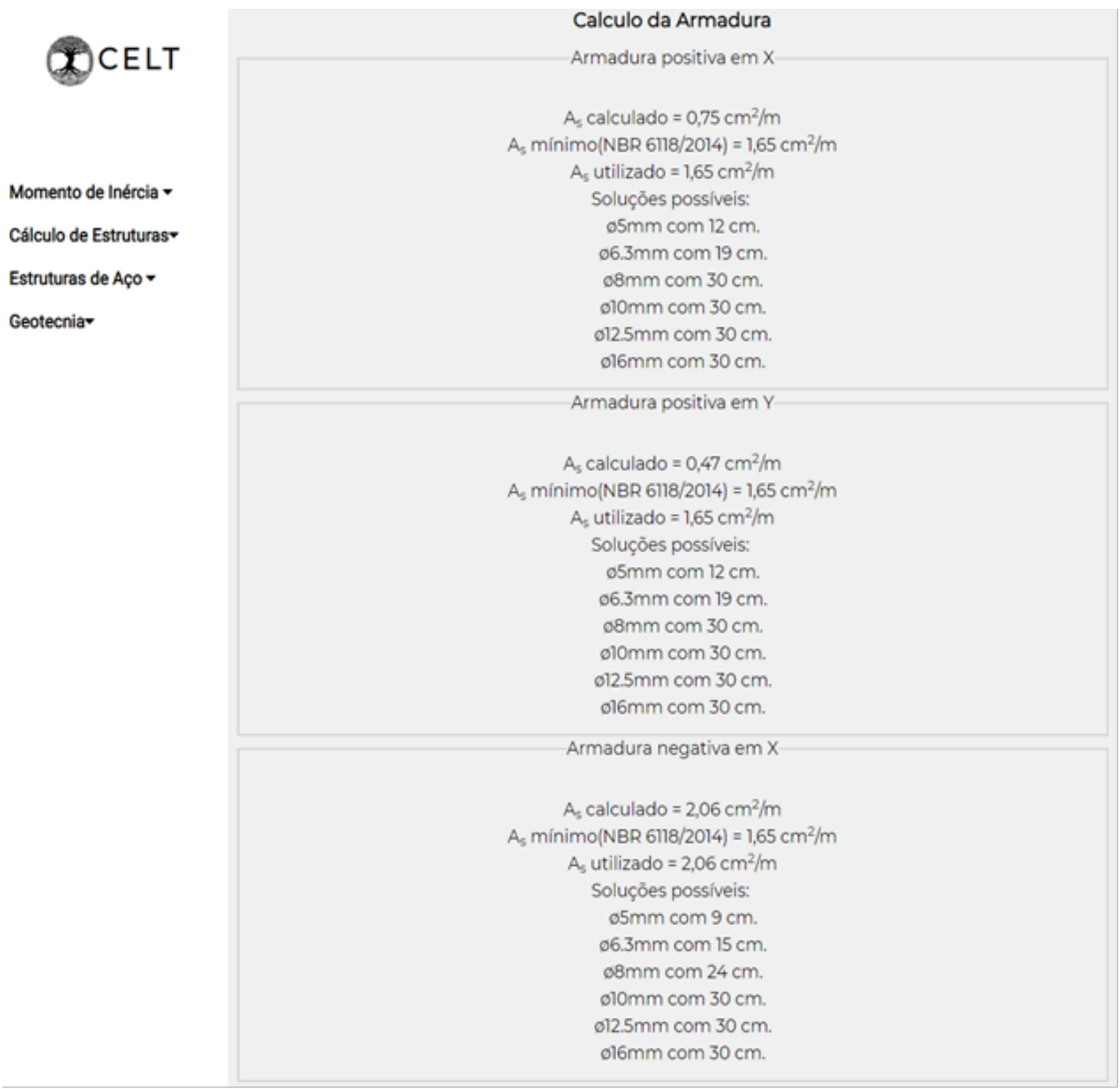

Fonte: (Autor, 2021)

Tabela 6: Dimensionamento da estrutura submetida a carga 2 pelo software

\begin{tabular}{c|c|c|c}
\hline & Direção X & Direção $Y$ & Engaste em $X$ \\
\hline Momentos $(\mathrm{kNm})$ & 6,5045 & 5,2976 & $-17,3829$ \\
\hline As $\left(\mathrm{cm}^{2} / \mathrm{m}\right)$ & 2,31 & 1,86 & 6,75 \\
\hline $\begin{array}{c}\text { As adotada } \\
\left(\mathrm{cm}^{2} / \mathrm{m}\right)\end{array}$ & 2,31 & 1,86 & 6,75 \\
\hline $\begin{array}{c}\text { Armadura } \\
\text { principal }\end{array}$ & $\varnothing 8 \mathrm{~mm}$ a cada $21 \mathrm{~cm}$. & $\varnothing 8 \mathrm{~mm}$ a cada $26 \mathrm{~cm}$. & $\emptyset 12.5 \mathrm{~mm}$ a cada $18 \mathrm{~cm}$. \\
\hline
\end{tabular}

Fonte: (Autor, 2021) 


\section{DISCUSSÃO}

A maior divergência de valores ocorreu no cálculo das áreas de armadura mínima, devido a utilização mínima da resistência do concreto de $20 \mathrm{MPa}$ pelo software, diferente dos $15 \mathrm{MPa}$ questionados pelo problema. Tal escolha se dá pelo uso cotidiano destas resistências maiores e, além do mais, dá-se o fato do exercício em questão preceder ao ano de 2002, estando submetido as condições tecnológicas da época. Mesmo assim, é possível observar que a relação dos resultados com a situação de entrada se mostra correta.

O programa, no momento de elaboração deste artigo, encontra-se em sua terceira atualização da versão básica. Primariamente, o mesmo realizava os cálculos por meio de tabelas, consultando as mesmas de acordo com o caso em que a laje se encontrava e retornando o valor desejado, e, nos casos não constantes nas tabelas, por meio de interpolação linear de valores vizinhos próximos. Em sua segunda atualização, foi adicionado o dimensionamento da armadura, por meio do mesmo método de consulta das tabelas de coeficientes k3 e k6. Na terceira e atual atualização, foi alterado método para o dimensionamento da laje, com a utilização direta de fórmulas para obtenção dos coeficientes utilizados na teoria das grelhas e a correção utilizada pelo método de Marcus, obtidos de Araújo (2010), além da adição do cálculo das reações nos apoios da laje, pelo método das linhas de ruptura. Esta última alteração só foi possível com uma revisão bibliográfica mais aprofundada, visto que, como observado pelo autor deste artigo, diversos autores procuram, por opção didática própria, a utilização de outros métodos, como os métodos de Bares ou Czerny, também amplamente difundidos, ou pela simples apresentação e utilização de tabelas para simplificação e maior eficiência do ensino do tema, sem aprofundar com tanta grandeza na teoria por trás dos mesmos.

\section{CONSIDERAÇÕES FINAIS}

Como demonstrado pelos resultados, o software é capaz de alcançar os mesmos resultados obtidos pelo cálculo manual, de forma rápida e completa, dentro do que se propõe. Tal praticidade se mostra importante, principalmente, no aprendizado do usuário, visto que permite a rápida verificação dos resultados e uma clareza na observação da relação das características da estrutura com os esforços e momentos gerados na mesma.

Além disso, por ser um software computacional, ele possui a vantagem de ser facilmente atualizado e elaborado para a integração de novas funcionalidades, como o cálculo conjunto de 
lajes contínuas de um pavimento; a adição do dimensionamento de vigas e pilares; a aplicação de novas metodologias de dimensionamento, dentre diversas outras. Junto a essa funcionalidade, também se agrega a sua praticidade de acesso e utilização, por não necessitar de instalação prévia e por poder ser utilizado em uma diversidade de dispositivos e equipamentos, sem necessidade de grande força computacional, apenas com o uso de uma conexão à internet.

Conclui-se, assim, que a integração tecnológica com a engenharia na educação é majoritária para o êxito em um ensino de excelência e coerente ao desenvolvimento, não só da profissão, mas para a sociedade como um todo.

\section{REFERÊNCIAS}

ARAÚJO, J.M. Curso de Concreto Armado, Vol. 2. 3ą. ed. Rio Grande, Editora DUNAS, 2010. ASSOCIAÇÃO BRASILEIRA DE NORMAS TÉCNICAS. NBR 6118: PROJETO DE ESTRUTURAS DE CONCRETO - PROCEDIMENTO. Rio de Janeiro, 2014.

BASTOS, P. S. dos S. Notas de Aula - Estruturas de Concreto I. São Paulo: UNESP, 2015. Disponível em: <http://wwwp.feb.unesp.br/pbastos/pag_concreto1.html>. Acesso em: 27 ago. 2019.

BOTELHO, M.H.C e MARCHETTI, O. Concreto armado, eu te amo. Vol.1., 5ạ edição. São Paulo, Edgard Blucher Ltda, 2008.

BOTELHO, M.H.C e MARCHETTI, O. Concreto armado, eu te amo. Vol.1., 3a edição. São Paulo, Edgard Blucher Ltda, 2002.

CLÍMACO, J.C.T.de S. Estruturas de concreto armado. Fundamentos de projeto, dimensionamento e verificação, 2008, 2a Edição. 